\title{
RNA and sex determination in Caenorhabditis elegans
}

\author{
Post-transcriptional regulation of the sex-determining tra-2 and fem-3 mRNAs in \\ the Caenorhabditis elegans hermaphrodite
}

\author{
Alessandro Puoti ${ }^{+}$, Paolo Pugnale, Marco Belfiore, Anne-Catherine Schläppi \& Zarifja Saudan \\ Department of Biology, University of Fribourg, Rue du Musée 10, 1700 Fribourg, Switzerland
}

Received April 10, 2001; revised August 9, 2001; accepted August 21, 2001

The Caenorhabditis elegans hermaphrodite sequentially produces sperm and oocytes from a single pool of precursors. Therefore, the hermaphrodite's germ line is the site of two major cell fate decisions: a germ cell precursor first undergoes a mitosis/meiosis decision and then a sperm/oocyte decision. While the mitosis/meiosis decision is governed by Notch/GLP-1 signalling, the sperm/oocyte decision relies on post-transcriptional regulation of two key mRNAs, tra-2 and fem-3. This review focuses on factors that are required for the silencing of these mRNAs, which results in the sequential production of sperm and oocytes. Most factors that regulate the expression of tra-2 and $f e m-3$ are homologous to proteins involved in RNA regulation in yeast, mammals or Drosophila, suggesting that at least some of the molecular mechanisms regulating the two worm mRNAs have been conserved throughout evolution.

\section{Introduction}

During the last two decades, numerous examples have shown that post-transcriptional regulation of gene expression is important in many organisms, particularly during embryogenesis and larval development (Wickens et al., 2000). Post-transcriptional regulation can occur at any step between transcription and translation. Thus it can affect RNA processing, RNA transport, stability or translation initiation. Post-transcriptional control not only allows a quicker response to the environment compared with de novo transcription, but also permits regulated gene expression in the absence of transcription (Mathews et al., 2000). This may be particularly important during early embryogenesis, when transcription is often repressed. Furthermore, regulation at the RNA level allows precise control of protein dosage, as is the case for mammalian tumour necrosis factor- $\alpha$ and Caenorhabditis elegans xol-1, which are regulated both transcriptionally and post-transcriptionally (Han et al., 1991; Nicoll et al., 1997).

Post-transcriptional regulation has been studied in many organisms, including $C$. elegans, in which several genes that are post-transcriptionally regulated have been identified. These genes code for the heterochronic RNAs lin-14, lin-28 and lin-41 (Lee et al., 1993; Moss et al., 1997; Slack et al., 2000), the xol-1 RNA that is responsible for primary sex determination (Nicoll et al., 1997; Skipper et al., 1999), the maternal RNAs glp-1 and apx-1 that are required for embryonic polarity (Evans et al., 1994; Mickey et al., 1996) and the germline sex determination fem-3 and tra-2 mRNAs (feminization; sexual transformer; Ahringer and Kimble, 1991; Goodwin et al., 1993). This review focuses on insights recently gained from studies on germline cell fate decisions mediated by the regulation of the tra- 2 and fem-3 RNAs whose products play distinct roles in specifying gametes as sperm or oocytes. In both cases, regulation is achieved via elements that are present in the $3^{\prime}$ untranslated regions (UTRs) of the RNAs.

Caenorhabditis elegans exists in two sexual forms, males and hermaphrodites. Hermaphrodites develop through four larval stages before reaching adulthood. They produce their first germ cells during the fourth larval stage (L4), and these differentiate into sperm. Approximately 160 sperm are made in each of the two gonadal arms at this time. After the L4-to-adult molt, spermatogenesis is turned off and all of the gametes that form subsequently develop into oocytes (for a review see Schedl, 1997). The sequential production of two fundamentally different germ cells requires post-transcriptional repression of two 


\section{review}

A. Puoti et al.
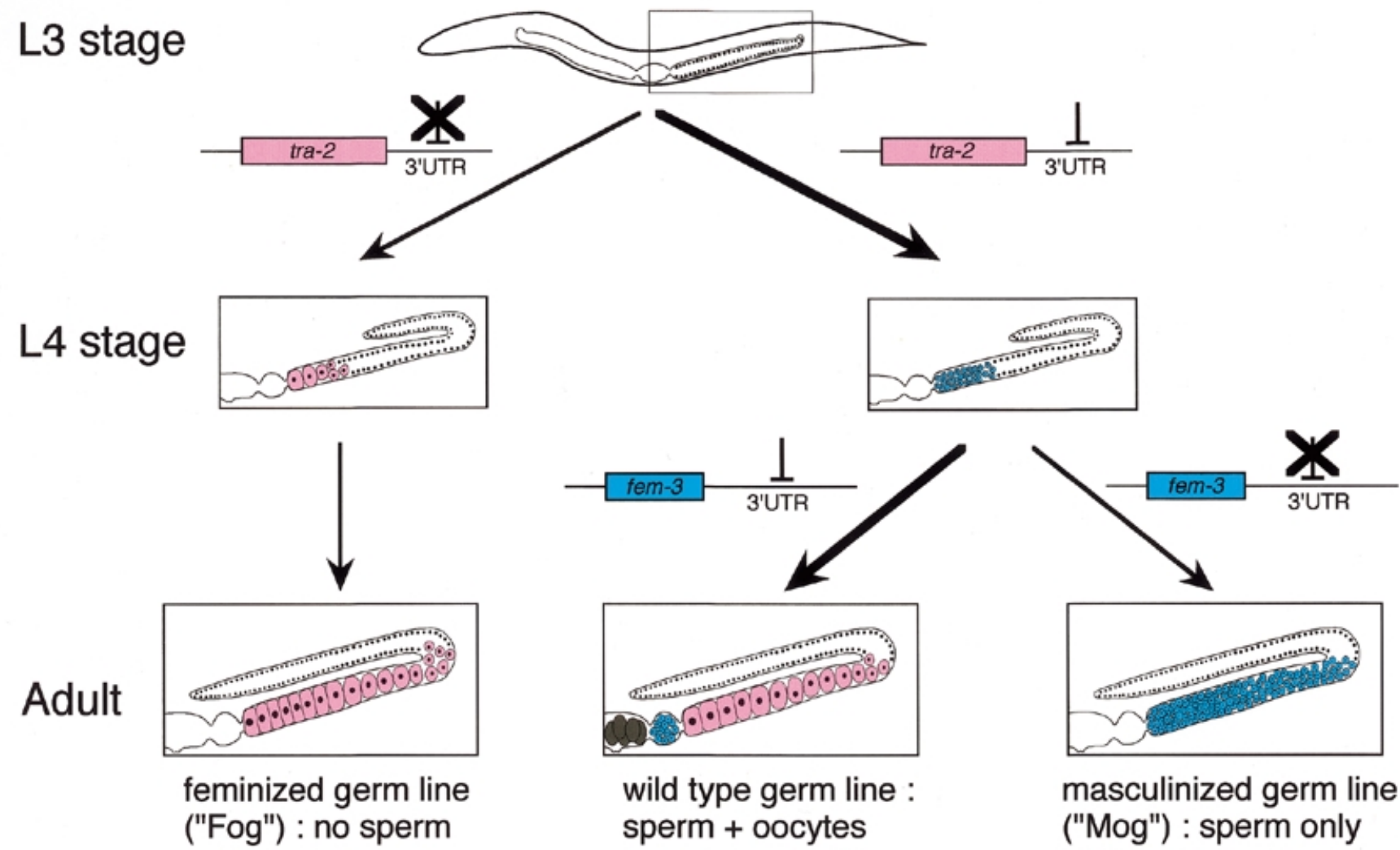

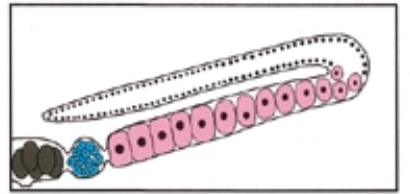

wild type germ line : sperm + oocytes

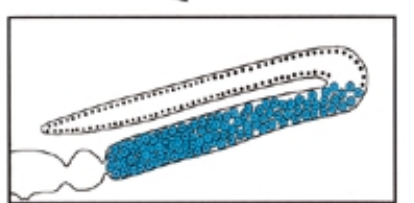

masculinized germ line ("Mog") : sperm only

Fig. 1. Development of the germ line in wild-type and mutant $C$. elegans hermaphrodites. The hermaphroditic gonad consists of two arms that extend during development and are connected through the vulva (top). The L3 worm is outlined with its anterior to the left. The right gonadal arm is boxed and shown to scale in L4 larvae and adults. Undifferentiated germ cell nuclei are shown as black dots, while mature sperm and oocytes are shown in blue and pink, respectively. Development of a wild-type hermaphrodite follows the thick arrows. Repression is shown by an inverted T. Suppression of repression is shown by a crossed inverted T. During the L3 stage, no mature gametes are present and the gonad arms start to reflex (top). At L4, wild-type hermaphrodites produce sperm (right) while tra-2 ( $g f$ ) mutants make oocytes (left). The onset of spermatogenesis is dependent on repression of tra-2 through its $3^{\prime} \mathrm{UTR}$. Wild-type adults contain both sperm and oocytes because spermatogenesis switches to oogenesis after the L4-to-adult molt (bottom, centre). This switch requires repression of the fem-3 mRNA through its 3'UTR. While tra-2 $(g f)$ mutants continue oogenesis, leading to a feminized germ line (Fog, bottom, left), fem-3 ( $g f)$ mutants do not switch to oogenesis and accumulate excess sperm (Mog, bottom, right).

mRNAs, tra-2 and fem-3; the former must be repressed for spermatogenesis, and the latter for oogenesis (Figure 1). While TRA-2 is a large transmembrane protein that has some similarity to the Patched receptor (Kuwabara and Kimble, 1995), no functional motifs have been found in the FEM-3 protein. One model proposes that FEM-3 is a cytoplasmic protein that binds to the intracellular portion of membrane-bound TRA-2 (Mehra et al., 1999) and that the release of FEM-3 from TRA-2 in response to the extracellular ligand HER-1 results in male development (Kuwabara and Kimble, 1992). In this model, the sperm/oocyte switch depends on the physical interactions between the TRA-2 and FEM-3 proteins and thus requires a finely tuned balance between TRA-2 and FEM-3. Regulation at the RNA level might be critical to achieving the proper stoichiometry.

\section{Repression of $t r a-2$ for the onset of spermatogenesis}

Genetic studies have shown that tra-2 promotes female development in that tra-2 loss-of-function (If) mutations transform XX animals into non-mating pseudomales that do not produce oocytes (Hodgkin and Brenner, 1977). Insight into tra-2 regulation came from analysis of tra-2 gain-of-function $(g f)$ mutants that do not produce sperm during L4, but instead produce only oocytes (Doniach, 1986; Figure 1). tra-2 expression is regulated by two repeated elements in the $3^{\prime} U T R$ that were identified through dominant tra-2 ( $g f$ ) mutations. These elements, the TGEs (for tra-2 and Gli Elements, formerly named DREs), consist of two repeats of 28 nucleotides that are separated by a fournucleotide spacer and that are disrupted by small deletions in tra-2 (gf ) alleles (Goodwin et al., 1993). Single copies of TGEs are also found in the $3^{\prime}$ UTR of other mRNAs such as the tra-2 mRNA from Caenorhabditis briggsae and the human oncogene $G L I$, suggesting that regulation through the TGE is conserved in other species (Jan et al., 1997).

Factors that bind directly to the TGEs or that negatively regulate tra-2 have been sought and have provided insights into the mechanisms that control tra-2 expression. RNA gel shift experiments performed with crude worm extracts revealed a binding activity-the direct repeat factor (DRF)—which specifically 
recognizes one TGE in the tra-2 3'UTR (Goodwin et al., 1993). DRF is a good candidate for a post-transcriptional repressor of tra-2, and its components are being characterized. These potentially include the GLD-1 (germ line development) protein, which was identified in a yeast three-hybrid screen as a specific binding factor for the TGEs (Jan et al., 1999). In addition, the F-box-containing protein FOG-2 (feminization of the germ line) was found to interact directly with GLD-1 and has been proposed to act as a bridge to assemble a tra-2 mRNA-multiprotein complex, which could repress tra-2 translation (Clifford et al., 2000). GLD-1, a cytoplasmic protein that belongs to the STAR (signal transduction and activation of $\underline{R} N A$ ) family has a single KH RNA-binding motif that is required for inhibition of tra-2 translation through the TGEs (Figure 2A; Jan et al., 1999). Consistent with their role in regulating tra-2 activity, GLD-1 and FOG-2 are both required for spermatogenesis and are expressed in the hermaphrodite germ line (Jones and Schedl, 1995; Clifford et al., 2000).

In another approach, a genetic screen for loss-of-function alleles that mimic a Tra-2 ( $g f$ ) phenotype identified the laf-1

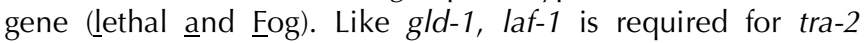
repression and works through the tra-2 3'UTR. laf- 1 is needed for spermatogenesis and for the repression, in vivo and in vitro, of transgenes bearing TGEs in their 3'UTRs (Goodwin et al., 1997). In addition, double mutant analyses indicate that laf- 1 acts upstream of tra-2, as would be predicted for a regulator of tra-2. The molecular identity of laf- 1 is not known yet, and hence direct binding of LAF-1 to the tra-2 3'UTR has not been tested. Future experiments will determine whether or not LAF-1 is another component of DRF.

Although polyribosome analysis has shown that tra-2 is regulated at the level of translation, and that repression via its $3^{\prime}$ UTR acts through the TGE by shortening of the tra- 2 mRNA poly $(\mathrm{A})$ tail (Thompson et al., 2000), the precise action of DRF on tra-2 remains to be elucidated.

In addition to regulation at the RNA level, the activity of TRA-2 might also be controlled post-translationally. In fact, tra-3 has been shown to encode a calpain-like protease that cleaves TRA-2 and generates a peptide with a feminizing activity that could bind and inactivate FEM-3 (Barnes and Hodgkin, 1996; Goodwin et al., 1997; Sokol and Kuwabara, 2000).

\section{Repression of $f e m-3$ for the sperm/oocyte switch}

fem-3 is necessary for spermatogenesis in both the male and the hermaphrodite; fem-3(If) mutants never produce sperm (Kimble et al., 1984). Conversely, fem-3(gf) mutants do not switch to oogenesis and thus accumulate excess sperm (Barton et al., 1987). These findings indicate that fem-3 inhibition is required for the sperm/oocyte switch.

The first evidence that fem-3 might be regulated at the RNA level came from fem-3(gf) mutants that were obtained as extragenic suppressors of fem-1(If) and fem-2(If) (Barton et al., 1987). The molecular lesions in all $19 \mathrm{fem}-3(\mathrm{gf})$ alleles are point mutations or small deletions in the 3'UTR, and disrupt a five-nucleotide element named the PME (point mutation element; Ahringer and Kimble, 1991). Thus, the PME may act as a cis-acting repressor of fem-3. In addition to its localization in the 3'UTR, three additional lines of evidence argue that the PME is respon-
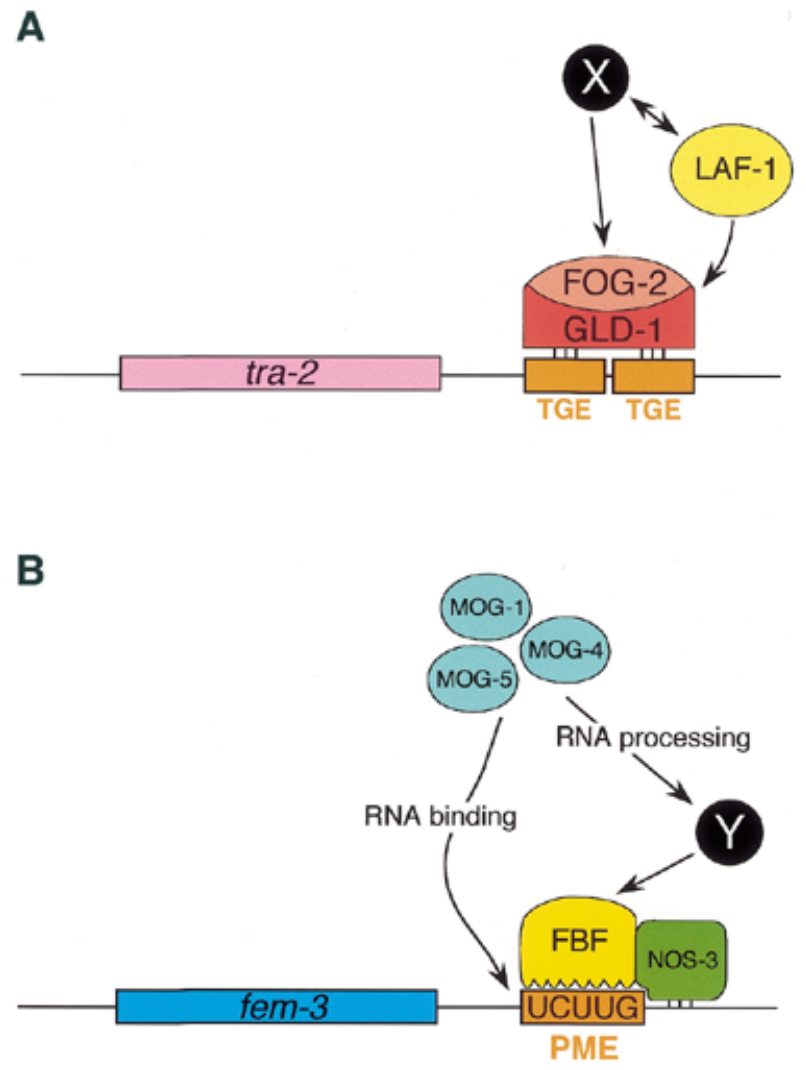

Fig. 2. Trans-acting regulators of tra-2 and fem-3. (A) tra-2 regulation. The GLD-1 protein binds specifically to the TGEs in the 3'UTR of the tra-2 mRNA. FOG-2 interacts with RNA bound to GLD-1. Although a single GLD-1 protein is shown here, the TGEs could be bound by several molecules of GLD-1. LAF-1 is an additional factor that regulates tra-2 either by binding directly to tra- 2 or by acting on GLD-1 or another putative factor (X). Some or all of the trans-acting factors that regulate tra-2 might form a protein complex named DRF. (B) fem-3 regulation. The model shows FBF and its interacting partner NOS-3 forming a cytoplasmic complex on the fem-3 mRNA. FBF specifically recognizes the PME through its eight Puf repeats, while NOS-3 binds RNAs non-specifically. Although the function of NOS-3 is redundant with those of NOS-1 and NOS-2, the latter proteins have not been included in this complex since they do not bind to FBF. On the other hand, at least three nuclear MOG proteins might act on fem-3 either directly or indirectly, via the FBF/NOS-3 complex and/or additional factors (Y). This action may involve RNA processing and RNA binding.

sible for the post-transcriptional rather than transcriptional regulation of fem-3. First, the steady-state levels of fem-3 mRNA are similar in wild-type animals and fem-3(gf) mutants. Secondly, a fem-3 RNA-binding activity can be titrated by expressing a heterologous RNA that contains the wild-type fem-3 3'UTR, resulting in masculinized germ lines in hermaphrodites. Such masculinization does not occur if a gain-of-function mutated fem-3 3'UTR is used, indicating that the wild-type fem-3 3'UTR is specifically bound by one or several factors that are required for the sperm/oocyte switch (Ahringer and Kimble, 1991). Thirdly, the somatic expression of a reporter construct bearing a heat shock promotor, the lac $Z$ gene and the $3^{\prime}$ UTR of fem-3 is normally repressed in wild-type worms, but it is de-repressed if a mutation disrupts the PME in the transgene (Gallegos et al., 


\section{review}

\section{A. Puoti et al.}

1998). In contrast to the distribution of tra-2, that of fem-3 mRNA in polyribosomes has not been studied so far, and as a consequence, it remains to be shown whether fem-3 is actually regulated at the level of RNA processing, nuclear export, stabilization or translation. Furthermore, good antibodies against TRA-2 and FEM-3 have not been obtained so far. Thus, it has not been shown that the protein levels of TRA-2 and FEM-3 actually change during gametogenesis.

Factors that act in trans to fem-3 via its $3^{\prime}$ UTR and that are required for the regulation of the sperm/oocyte switch have been sought via both genetic and biochemical means. The rationale behind the genetic screens that were undertaken was that, if such trans-acting factors are required for fem-3 repression, a mutation leading to the loss of the repressor should generate a phenotype that is similar to Fem-3(gf). Genetic screens identified six mog genes (masculinization of the germ line) that act upstream of fem-3 and are required for the sperm/oocyte switch in hermaphrodites (Graham and Kimble, 1993; Graham et al., 1993). Additional evidence for mog function in fem-3 regulation came from the observation that protein expression from the lacZ::fem-3 3'UTR transgene was higher in each of the six mog mutants than in wild-type worms, indicating that the mog genes are required for fem-3 repression through its $3^{\prime} \mathrm{UTR}$ in vivo (Gallegos et al., 1998). Of the six mog genes, mog-1, mog-4 and mog-5 were cloned and found to encode nuclear proteins of a particular DEAH-box RNA helicase subfamily. These proteins are the $C$. elegans homologues of yeast splicing factors Prp16p, Prp2p and Prp22p, respectively, suggesting that fem-3 regulation could involve pre-mRNA splicing as well (Puoti and Kimble, 1999, 2000).

In addition to the genetic approach, yeast two- and threehybrid screens were used to identify trans-acting factors that either bind to known fem-3 regulators via protein-protein interactions or that bind directly to the fem-3 $3^{\prime} U T R$, respectively. To identify factors that bind specifically to the fem-3 3'UTR, Zhang et al. (1997) used a yeast three-hybrid screen for RNA-binding proteins. Specifically, two 37-nucleotide tandem repeats containing the PME were used as RNA bait. This screen yielded two distinct proteins, FBF-1 and FBF-2 (fem-3 binding factor), which share $91 \%$ amino acid identity and that are probably redundant. RNA interference directed against both fbf- 1 and $f b f-2$ led to masculinized hermaphrodites that accumulate excess sperm and do not switch to oogenesis, suggesting that $f b f$ is required for the switch from spermatogenesis to oogenesis (Zhang et al., 1997).

In another approach, protein-protein interaction screens using the yeast two-hybrid system identified additional trans-acting factors. The fem-3-binding protein FBF-1 was used as a bait for interacting proteins (Kraemer et al., 1999). The C. elegans protein NOS-3 (Nanos) was identified as a binding partner for both FBF-1 and FBF-2 in the yeast two-hybrid system and in vitro. NOS-3 binds directly to FBF proteins but has an additional general RNA-binding activity that is not required for its binding to FBF. NOS-3 contains two unusual zinc fingers in its C-terminus and has at least two orthologues in C. elegans, NOS-1 and NOS-2, which are required for germ-line viability (Subramaniam and Seydoux, 1999). Similarly to FBF, the three nos genes are likely to have redundant functions since sterility or occasional excess sperm was only observed in nos double or triple mutants (Kraemer et al., 1999; Subramaniam and Seydoux, 1999).

\section{FBF-like and NOS-like repressors}

FBF is a member of a large protein family that is characterized by the presence of eight Puf (Pumilio and EBF) repeats of $\sim 37$ amino acids and its Drosophila counterpart is the Pumilio (Pum) protein (Zhang et al., 1997). While FBF represses fem-3 expression by acting on the fem-3 3'UTR, Pum is necessary to repress the translation of the hunchback $(h b)$ mRNA through binding to the NREs (Nanos response elements), which are located in the $h b 3^{\prime}$ UTR (Wharton and Struhl, 1991). Repression of $h b$ involves deadenylation of the mRNA and requires the formation of a protein complex that includes at least Pum, Nanos (Nos) and the NREs (Wharton and Struhl, 1991; Wreden et al., 1997). In Drosophila, the interaction between Nos and Pum is dependent on the presence of the $h b$ mRNA (Sonoda and Wharton, 1999). Additional components such as the Drosophila 'Brain Tumor' (Brat) protein, which forms a quaternary complex by associating with $h b$ RNAbound Pum and Nos (Sonoda and Wharton, 2001), might be required for the repression of $h b$ mRNA. Intriguingly, one Brat homologue in $C$. elegans is LIN-41, which is required for the switch from $\mathrm{L} 4$ to adult and which post-transcriptionally regulates the heterochronic mRNA lin-29 (Slack et al., 2000). Drosophila nos encodes a general RNA-binding protein with two unusual $\mathrm{CCHC}$ zinc fingers that are conserved in the $C$. elegans NOS-1, NOS-2 and NOS-3 proteins. Unlike Drosophila Nos and Pum, C. elegans FBF and NOS-3 associate even in the absence of an RNA molecule. Moreover, no interaction has so far been reported between LIN-41 and either FBF or NOS-3. However, the sequence similarities between NOS-3 and Drosophila Nos and their cooperation with FBF and Pum, respectively, suggest that similar molecular mechanisms govern fem- 3 and $h b$ repression.

Drosophila Nos was first discovered to be essential, together with Pum, for posterior patterning of the Drosophila embryo (Lehmann and Nusslein-Volhard, 1991), while FBF and NOS-3 were identified as regulators of fem-3 and hence implicated in the sperm/oocyte switch in $C$. elegans hermaphrodites. However, recent studies have shown that Pum is also required for germ cell development and migration in the embryo and for maintenance of the germline stem cells in the adult ovary (Forbes and Lehmann, 1998; Asaoka-Taguchi et al., 1999). Similarly, the $C$. elegans nos and $f b f$ genes might also have functions beyond those in the sperm/oocyte switch since nos- 1 to -3 , and some $C$. elegans pum homologues including $f b f$, appear to be required for the proliferation and survival of germ cells (Kraemer et al., 1999; Subramaniam and Seydoux, 1999).

Concerning cis-acting elements, Tadauchi et al. (2001) have shown that Mpt5p, a Puf family member in Saccharomyces cerevisiae also regulates mRNA through $3^{\prime} U T R$ elements, in this case, that of the $\mathrm{HO}$ mating type switch gene. Like Pum, yeast Mpt5p negatively regulates $H O$ by destabilizing the mRNA, perhaps by promoting its deadenylation (Tadauchi et al., 2001). Interestingly, sequence conservation is also found in the $3^{\prime}$ UTRs of fem-3, $h b$ and $H O$ mRNAs. In fact a four-nucleotide sequence (UUGU) is found twice in each NRE, once in the HO 3'UTR and once in the fem-3 3'UTR, where it almost overlaps with the PME (ucUUGU; Zamore et al., 1997; Tadauchi et al., 2001). Together with the similarities found between Puf family members and their interaction with Nos-like proteins, the identification of a partially conserved cis-acting element 
suggests that Puf/Nos-dependent RNA regulation may represent an ancient mechanism for post-transcriptional regulation of gene expression.

\section{Future perspectives}

The cis-acting regulatory elements in fem-3 and tra-2 were discovered 10 years ago. At present, several factors that are required for the regulation of tra- 2 and fem-3 are known, and their initial characterization indicates that the regulation of the two mRNAs in C. elegans involves trans-acting factors and cisacting elements that may have similar or related functions in other species. Although a molecular function has been proposed for most of the trans-acting regulators that have been identified, the molecular mechanisms through which they regulate tra-2 or fem-3 are not well understood. Searches for additional components, the establishment of in vitro systems, and comparisons with other model systems such as Drosophila and yeast are likely to bring the answers.

\section{Acknowledgements}

We thank Betsy Goodwin and Adrian Streit for comments on the manuscript. Thanks to $\mathrm{H}$. Gachoud for preparing the figures. A.P. is supported by grants 3130-054989.98 and 3100-055384.98 from the Swiss National Science Foundation.

\section{References}

Ahringer, J. and Kimble, J. (1991) Control of the sperm-oocyte switch in Caenorhabditis elegans hermaphrodites by the fem-3 3'untranslated region. Nature, 349, 346-348.

Asaoka-Taguchi, M., Yamada, M., Nakamura, A., Hanyu, K. and Kobayashi, S. (1999) Maternal Pumilio acts together with Nanos in germline in Drosophila embryos. Nature Cell Biol., 1, 431-437.

Barnes, T.M. and Hodgkin, J. (1996) The tra-3 sex determination gene of Caenorhabditis elegans encodes a member of the calpain regulatory protease family. EMBO J., 17, 4477-4484.

Barton, M.K., Schedl, T.B. and Kimble, J. (1987) Gain-of-function mutations of fem-3, a sex-determination gene in Caenorhabditis elegans. Genetics, 115, 107-119.

Clifford, R., Lee, M.-H., Nayak, S., Ohmachi, M., Giordini, F. and Schedl, T. (2000) FOG-2, a novel F-box containing protein, associates with the GLD-1 RNA binding protein and directs male sex determination in the C. elegans hermaphrodite. Development, 127, 5265-5276.

Doniach, T. (1986) Activity of the sex-determining gene tra-2 is modulated to allow spermatogenesis in the C. elegans hermaphrodite. Genetics, 114, 53-76.

Evans, T.C., Crittenden, S., Kodoyianni, V. and Kimble, J. (1994) Translational control of maternal $g l p-1$ mRNA establishes an asymmetry in the C. elegans embryo. Cell, 77, 183-194.

Forbes, A. and Lehmann, R. (1998) Nanos and Pumilio have critical roles in the development and function of Drosophila germline stem cells. Development, 125, 679-690.

Gallegos, M., Ahringer, J., Crittenden, S. and Kimble, J. (1998) Repression by the 3' UTR of $f e m-3$, a sex-determining gene, relies on a ubiquitous mog-dependent control in Caenorhabditis elegans. EMBO J., 17, 6337-6347.

Goodwin, E.B., Okkema, P.G., Evans, T.C. and Kimble, J. (1993) Translational regulation of tra-2 by its $3^{\prime}$ untranslated region controls sexual identity in C. elegans. Cell, 75, 329-339.

Goodwin, E.B., Hofstra, K., Hurney, C.A., Mango, S. and Kimble, J. (1997) A genetic pathway for regulation of tra-2 translation. Development, 124, 749-758.
Graham, P.L. and Kimble, J. (1993) The mog- 1 gene is required for the switch from spermatogenesis to oogenesis in Caenorhabditis elegans. Genetics, 133, 919-931.

Graham, P.L., Schedl, T. and Kimble, J. (1993) More mog genes that influence the switch from spermatogenesis to oogenesis in the hermaphrodite germ line of Caenorhabditis elegans. Dev. Genet., 14, 471-484.

Han, J., Beutler, B. and Huez, G. (1991) Complex regulation of tumor necrosis factor mRNA turnover in lipopolysaccharide-activated macrophages. Biochim. Biophys. Acta, 1090, 22-28.

Hodgkin, J.A. and Brenner, S. (1977) Mutations causing transformation of sexual phenotype in the nematode Caenorhabditis elegans. Genetics, 86, 275-287.

Jan, E., Yoon, J.W., Walterhouse, D., Iannaccone, P. and Goodwin, E.B. (1997) Conservation of the C. elegans tra-2 3'UTR translational control. EMBO J., 16, 6301-6313.

Jan, E., Motzny, C.K. and Graves, L.E. (1999) The STAR protein, GLD-1, is a translational regulator of sexual identity in Caenorhabditis elegans. EMBO J., 18, 258-269.

Jones, A.R. and Schedl, T. (1995) Mutations in gld-1, a female germ cellspecific tumor suppressor gene in Caenorhabditis elegans, affect a conserved domain also found in Src-associated protein Sam68. Genes Dev., 9, 1491-1504.

Kimble, J., Edgar, L. and Hirsh, D. (1984) Specification of male development in Caenorhabditis elegans: the fem genes. Dev. Biol., 105, 234-239.

Kraemer, B., Crittenden, S., Gallegos, M., Moulder, G., Barstead, R., Kimble, J. and Wickens, M. (1999) NANOS-3 and FBF proteins physically interact to control the sperm-oocyte switch in Caenorhabditis elegans. Curr. Biol., 9, 1009-1018.

Kuwabara, P.E. and Kimble, J. (1992) Sex determination in Caenorhabditis elegans. J. Nematol., 24, 324-329.

Kuwabara, P.E. and Kimble, J. (1995) A predicted membrane protein, TRA2A, directs hermaphrodite development in Caenorhabditis elegans. Development, 121, 2995-3004.

Lee, R.C., Feinbaum, R.L. and Ambros, V. (1993) The C. elegans heterochronic gene lin-4 encodes small RNAs with antisense complementarity to lin-14. Cell, 75, 843-854.

Lehmann, R. and Nusslein-Volhard, C. (1991) The maternal gene nanos has a central role in posterior pattern formation of the Drosophila embryo. Development, 112, 679-691.

Mathews, M.B., Sonenberg, N. and Hershey, J.W.B. (2000) Origins and principles of translational control. In Sonenberg, N., Hershey, J.W.B. and Mathews, M.B. (eds), Translational Control of Gene Expression. Cold Spring Harbor Laboratory Press, Cold Spring Harbor, NY, pp. 1-31.

Mehra, A., Gaudet, J., Heck, L., Kuwabara, P.E. and Spence, A.M. (1999) Negative regulation of male development in Caenorhabditis elegans by a protein-protein interaction between TRA-2A and FEM-3. Genes Dev., 13, 1453-1463.

Mickey, K.M., Mello, C.C., Montgomery, M.K., Fire, A. and Priess, J.R. (1996) An inductive interaction in 4-cell stage $C$. elegans embryos involves APX-1 expression in the signalling cell. Development, 122, 1791-1798.

Moss, E.G., Lee, R.C. and Ambros, V. (1997) The cold shock domain protein LIN-28 controls developmental timing in C. elegans and is regulated by the lin-4 RNA. Cell, 88, 637-646.

Nicoll, M., Akerib, C.C. and Meyer, B.J. (1997) X-chromosome-counting mechanisms that determine nematode sex. Nature, 388, 200-204.

Puoti, A. and Kimble, J. (1999) The Caenorhabditis elegans sex determination gene mog- 1 encodes a member of the DEAH-Box protein family. Mol. Cell. Biol., 19, 2189-2197.

Puoti, A. and Kimble, J. (2000) The hermaphrodite sperm/oocyte switch requires the Caenorhabditis elegans homologs of PRP2 and PRP22. Proc. Natl Acad. Sci. USA, 97, 3276-3281.

Schedl, T. (1997) Developmental genetics of the germ line. In Riddle, D.L., Blumenthal, T., Meyer, B.J. and Priess, J.R. (eds), 


\section{review}

\section{A. Puoti et al.}

C. elegans II. Cold Spring Harbor Laboratory Press, Cold Spring Harbor, NY, pp. 241-269.

Skipper, M., Milne, C.A. and Hodgkin, J. (1999) Genetic and molecular analysis of fox-1, a numerator element involved in Caenorhabditis elegans primary sex determination. Genetics, 151, 617-631.

Slack, F.J., Basson, M., Liu, Z., Ambros, V., Horvitz, H.R. and Ruvkun, G. (2000) The lin-41 RBCC gene acts in the C. elegans heterochronic pathway between the let-7 regulatory RNA and the LIN-29 transcription factor. Mol. Cell, 5, 659-669.

Sokol, S.B. and Kuwabara, P.E. (2000) Proteolysis in Caenorhabditis elegans sex determination: cleavage of TRA-2 by TRA-3. Genes Dev., 14, 901-906.

Sonoda, J. and Wharton, R.P. (1999) Recruitment of Nanos to hunchback mRNA by Pumilio. Genes Dev., 13, 2704-2712.

Sonoda, J. and Wharton, R. (2001) Drosophila Brain Tumor is a translational repressor. Genes Dev., 15, 762-773.

Subramaniam, K. and Seydoux, G. (1999) nos-1 and nos-2, two genes related to Drosophila nanos, regulate primordial germ cell development and survival in Caenorhabditis elegans. Development, 126, 4861-4871.

Tadauchi, T., Matsumoto, K., Herskowitz, I. and Irie, K. (2001) Posttranscriptional regulation through the $H O 3^{\prime}$-UTR by $\mathrm{Mpt5}$, a yeast homolog of Pumilio and FBF. EMBO J., 20, 552-561.

Thompson, S.R., Goodwin, E.B. and Wickens, M. (2000) Rapid deadenylation and poly (A)-dependent translational repression mediated by the Caenorhabditis elegans tra-2 3' untranslated region in Xenopus embryos. Mol. Cell. Biol., 20, 2129-2137.

Wharton, R.P. and Struhl, G. (1991) RNA regulatory elements mediate control of Drosophila body pattern by the posterior morphogen nanos. Cell, 67, 955-967.

Wickens, M., Goodwin, E.B., Kimble, J., Strickland, S. and Hentze, M.W. (2000) Translational control of developmental decisions. In Sonenberg, N., Hershey, J.W.B. and Mathews, M.B. (eds), Translational Control of Gene Expression. Cold Spring Harbor Laboratory Press, Cold Spring Harbor, NY, pp. 295-370.

Wreden, C., Verotti, A.C., Schisa, J.A., Lieberfarb, M.A. and Strickland, S. (1997) Nanos and pumilio establish embryonic polarity in Drosophila by promoting posterior deadenylation of hunchback mRNA. Development, 124, 3015-3023.

Zamore, P.D., Williamson, J.R. and Lehmann, R. (1997) The Pumilio protein binds RNA through a conserved domain that defines a new class of RNAbinding proteins. RNA, 3, 1421-1433.

Zhang, B., Gallegos, M., Puoti, A., Durkin, E., Fields, S., Kimble, J. and Wickens, M.P. (1997) A conserved RNA-binding protein that regulates sexual fates in the C. elegans hermaphrodite germ line. Nature, 390, 477-484.

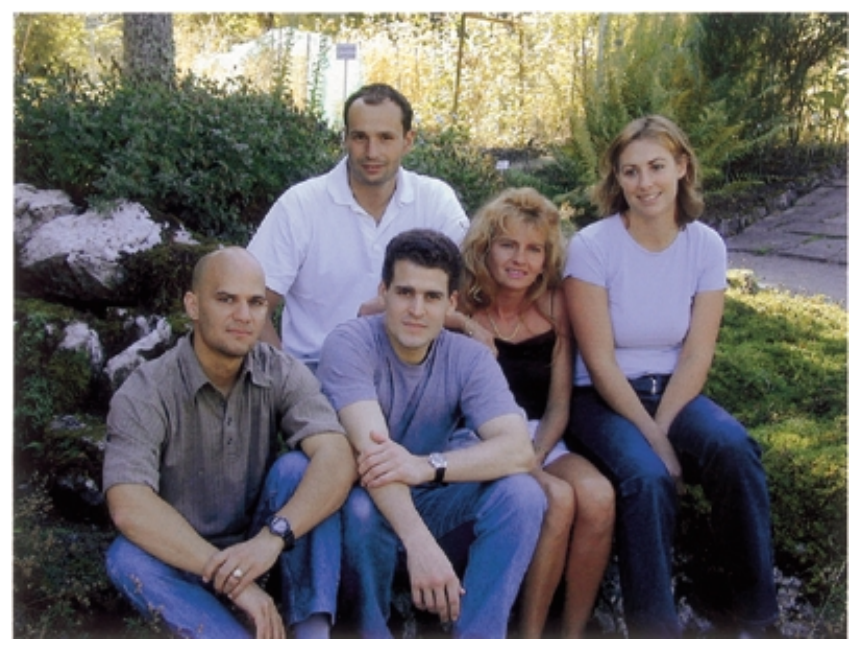

Front from left: Paolo Pugnale, Marco Belfiore, Zarifja Saudan \& Anne-Catherine Schläppi. Back in the centre: Alessandro Puoti

DOI: 10.1093/embo-reports/kve209 